\title{
Shannon information of the geomagnetic field for the past 7000 years
}

\author{
A. De Santis ${ }^{1,2}$ and E. Qamili ${ }^{1,3}$ \\ ${ }^{1}$ Istituto Nazionale di Geofisica e Vulcanologia, Roma, Italy \\ ${ }^{2}$ Facoltà di Scienze Matematiche, Fisiche e Naturali, Università degli Studi “G. D'Annunzio", Chieti, Italy \\ ${ }^{3}$ Scuola di dottorato in Scienze Polari, Università degli studi di Siena, Siena, Italy
}

Received: 25 March 2009 - Revised: 7 January 2010 - Accepted: 25 January 2010 - Published: 12 February 2010

\begin{abstract}
The present behaviour of the geomagnetic field as expressed by the International Geomagnetic Reference Field (IGRF) deserves special attention when compared with that shown over the past few thousands of years by two paleomagnetic/archeomagnetic models, CALS3K and CALS7K. The application of the Information theory in terms of Shannon Information and K-entropy to these models shows characteristics of an instable geomagnetic field. Although the result is mitigated when we correct the CALS7K model for its typical spectral damping, the present geomagnetic field as represented by IGRF is still rather distinct, at least for the past 4000 years, a result that is further confirmed by the CALS3K model. This is consistent with a significant global critical state started at around 1750, and still present, characterised by significant decays of the geomagnetic dipole, energy and Shannon information and high K-entropy. The details of how these characteristics may develop are not clear, since the present state could move toward an excursion or a geomagnetic polarity reversal, but we cannot exclude the possibility that the "critical" behaviour will become again more "normal", stopping the apparent trend of the recent geomagnetic field decay.
\end{abstract}

\section{Introduction}

Considerable interest has focused recently on how the present geomagnetic field is distinct from the field of the past several millennia. In particular, evidence is mounting that the present geomagnetic field may be approaching a geomagnetic reversal or major excursion (Gubbins, 1987; Hulot et al., 2002; De Santis et al., 2004) with significant possible implications in the biosphere, in the atmosphere and in

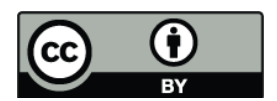

Correspondence to: A. De Santis (angelo.desantis@ingv.it) some other components of the Earth system (Korte and Constable, 2006). For example, Gubbins et al. (2006) estimated that the rate of decay of the geomagnetic field since 1840 was about $5 \%$ per century and also quite erratic. The conventional multipolar approach, i.e. the representation of the geomagnetic field potential in terms of the sum of the dipole and non-dipole terms, may not provide clear understanding of the dynamical and configurational properties of the whole field, because the analysis and its consequent results are usually obscured by the huge dipolar contribution with respect to the others. Therefore, other kinds of analyses must be exploited following a more holistic approach to the problem that looks at the field for its wholeness rather than at each specific and minute part (e.g. De Santis, 2009). Along this line of reasoning, De Santis et al. (2004) applied some concepts of Information Theory to the recent (last 100 years) geomagnetic field using the IGRF (International Geomagnetic Reference Field) model (Macmillan and Maus, 2005). De Santis $(2007,2008)$ extended this analysis to the geomagnetic field over the past 400 years using the GUFM1 geomagnetic field model (Jackson et al., 2000; validity 1590-1990), which is the best representation of the field in the instrumental period of geomagnetism, with particular emphasis on the records of the last 100 years. De Santis (2007) identified interesting features in some physical and statistical quantities to indicate that the geomagnetic field had been decaying for the past three centuries and likely will continue for few centuries more. In general, these results provide insights on geomagnetic field behaviour over only a very limited window of time. Archeomagnetic and paleomagnetic data offer opportunities for testing and significantly extending the results, even though the quality of the geomagnetic field intensity estimates from these data is not as good as instrumental observations and some cautions on their use for the purposes of this paper must be taken in due considerations (De Santis, 2007). In this study, we analyze the global geomagnetic field models CALS7K (Korte and Constable, 2005a, b, 2006) 
and CALS3K (Korte et al., 2009) with Gauss spherical harmonic coefficients that describe the geomagnetic field behaviour from 7000 and 3000 years ago, respectively, to the present. Following our previous approach (De Santis et al., 2004, De Santis, 2007), we evaluate these models for the behaviour of the Shannon information and related quantities (Shannon, 1948; Beck and Schlögl, 1993). In particular, we establish and compare the variation of the above mentioned quantities over the past 3000 and 7000 years as deduced from the CALS3K and CALS7K global models, respectively, to affirm that the present geomagnetic field behaviour is consistent with a current planetary transition in terms of a significant change of its main characteristics (energy, dipole moment, related core dynamics, etc.) possibly going to an excursion or even a reversal in progress. In the next sections, we outline the applications of the information theory to characterise the properties of the geomagnetic field, and also describe the CALS7K and CALS3K models. We do not analyse the secular variation because of the larger errors involved in the analysis (a partial example of this fact was given by the analysis in De Santis et al., 2004); in addition, regarding this aspect, we must recall that the equivalent source depth for the secular variation is not as clear as for the field (Holme and Olsen, 2005).

We then compare these results for the longer term CALS7K and CALS3K models with those of the shorter term IGRF model and discuss their implications for the present geomagnetic field decay.

\section{Shannon information and K-entropy of the geomagnetic field}

The concept of Information Content or Shannon Information $I(t)$ (Shannon, 1948; Beck and Schlögl, 1993) has been already defined and applied to the present geomagnetic field (De Santis et al., 2004; De Santis, 2007). The geomagnetic field $\mathbf{B}(t)$ can be defined at and above the Earth's surface as the negative gradient of a scalar potential $V(t)$, which can be, in turn, expressed at each time $t$ by a spherical harmonic (SH) expansion in space characterized by a set of Gauss coefficients $\left\{g_{n}^{m}(t), h_{n}^{m}(t)\right\}$ with $n=1, \ldots, N$ degrees and $m=0, \ldots, n$ orders of the expansion. This SH expansion represents the superposition of $N$ multipoles, and we can define the information content $I(t)$ of $\mathbf{B}(t)$ as (De Santis et al., 2004):

$I(t)=\sum_{n=1}^{N} p_{n}(t) \cdot \ln p_{n}(t)$,

with the probability, $p_{n}(t)$, for the $n$-th multipole given by:

$$
p_{n}=\frac{\left\langle\mathbf{B}_{n}^{2}\right\rangle}{\left\langle\mathbf{B}^{2}\right\rangle}=\frac{(n+1) q^{2 n+4} \sum_{m=0}^{n}\left(c_{n}^{m}\right)^{2}}{\sum_{n^{\prime}=1}^{N}\left(n^{\prime}+1\right) q^{2 n^{\prime}+4} \sum_{m=0}^{n^{\prime}}\left(c_{n^{\prime}}^{m}\right)^{2}},
$$

where $\left(c_{n}^{m}\right)^{2}=\left(g_{n}^{m}\right)^{2}+\left(h_{n}^{m}\right)^{2} ; q=a / r, a=6371.2 \mathrm{~km}$, and $\sum_{n} p_{n}=1$ so that $p_{n} \ln p_{n}=0$ if $p_{n}=0 .<\mathbf{B}^{2}>$ and $<\mathbf{B}_{n}^{2}>$ are the mean squared amplitudes over the sphere with radius $r$ of the total field and of the field due to the $n$-th multipole, respectively (Lowes, 1966; De Santis et al., 1995), which also correspond to the total and $n$-multipole contributions, respectively, to the spatial power spectrum of the geomagnetic field (Lowes, 1974). Our definition of probability follows that one usually given for spectral images analysis (Jernigan and D'Astous, 1984; Ryzhikov et al., 1995; RodriguesIturbe et al., 1998; Ferraro et al., 2002). Analogously, we could introduce the probability $p_{n}^{\prime}$ for the secular variation $(d \mathbf{B} / d t)=\dot{\mathbf{B}}$, as in (2) but using $\left(\dot{c}_{n}^{m}\right)^{2}=\left(\dot{g}_{n}^{m}\right)^{2}+\left(\dot{h}_{n}^{m}\right)^{2}$ instead of $\left(c_{n}^{m}\right)^{2}$.

In general, $I(t)$ is a negative quantity that measures the state of the system from only the probability distribution $p$ (Beck and Schlögl, 1993): the lower $I(t)$ the more complex is the spatial configuration at time $t$. It is simply related to the Shannon Entropy $H(\mathrm{t})$ by $I(t)=-H(t)$ (Beck and Schlögl, 1993; Majewski, 2001). In practice, $I(t)$ denotes our decreasing ability to predict the evolution of the system into the future, and thus the way $I(t)$ changes with time reflects the intrinsic dynamics of the system. To take into account this aspect, we can relate $I(t)$ with the rate of loss of information or the Kolmogorov entropy or K-entropy (Wales, 1991):

$\mathrm{K}=-\frac{d I}{d t}$

A chaotic process is characterised by a linear decay of $I$ with slope $-\mathrm{K}$ so that after time $T=1 / \mathrm{K}$, the system's behaviour can no longer be predicted. The geomagnetic field at the time of a reversal or excursion is characterized by having most of the coefficients $c_{n}^{m}$ nearly zero (e.g., Jacobs, 1994) so that $p_{n} \approx p_{n}^{\prime}$ and both K-entropies of the field and its secular variation must be similar. These information parameters for the present geomagnetic field are in general agreement with each other (De Santis et al., 2004). Thus, if these results reflect a real global transition in progress, with possible extreme outcome as a reversal or excursion, the characteristic time $T$ related to the duration of this possible phenomenon is rather short, between 400 and 800 years (De Santis et al., 2004).

To our knowledge, Eq.(3) is always valid point-to-point for chaotic processes. Actually, K-entropy measures the mean information loss during the evolution of the system between successive points on the trajectory in the phase space (e.g. Beck and Schlögl, 1993; Buchner and Zebrowski, 1998). Thus, for a more general case, the information quantities, in particular the probability used in (1) and the K-entropy, should be estimated in the phase space. But this is very difficult in our case, having a short time series of Gauss coefficients (if we consider a point every 5 years for IGRF, we have only around 20 points, and a point every 100 years for CALS3K and CALS7K, we have 30 and 70 points, respectively). However, we can suppose that the geomagnetic field 
is ergodic, i.e. time average of the original signal is equal to density average in the phase space (Eckmann and Ruelle, $1985)$, consequently we can resort to appropriate time averages. This is also confirmed by Baranger et al. (2002), who show that Eq. (3) is still valid when we average over many histories of the signal of concern. Therefore, we divide the whole time series of $I(t)$ as deduced from (1) in many successive temporal 100-year segments (we chose this interval because it is comparable with the time length of IGRF validity and it is the typical time uncertainty of the CALS3K and CALS7K models; see also below), then we make a linear fit over the corresponding $I(t)$ values and finally we estimate $\mathrm{K}$ as the (negative) slope of the linear fit, attributing the obtained value to the centre of each interval of time. When applied to IGRF the method is the same as it was already applied in De Santis et al. (2004).

\section{The CALS7K and CALS3K models}

The continuous global geomagnetic field model, CALS7K (Continuous Archeomagnetic and Lake Sediment model for the past $7 \mathrm{~K}$ years, v.2) attempts to account for the geomagnetic field behaviour from $5000 \mathrm{BC}$ to $1950 \mathrm{AD}$ (Korte and Constable, 2005a, b). It is based on a spatial spherical harmonic expansion up to degree and order 10 and on the temporal expansion in cubic B-splines of lake sediment paleomagnetic observations and archeomagnetic data (including lava flows), which include nearly 30000 inclination and declination values and 3188 intensity values. The data distribution was not homogeneous in space and time. For instance, the archeomagnetic data are biased to the Northern Hemisphere and Europe, whereas poor data coverage characterises the Southern Hemisphere.

The model was determined by regularized least squares inversion of these datasets. In the time of validity of CALS7K, periods of weak dipole moment and spatial power are evident (Korte and Constable, 2005a, b). In the overlapping period of validity with the latest IGRF, i.e., from 1900 to 1950, there is a general agreement with the corresponding geomagnetic field values. Although the errors associated with the archeomagnetic and paleomagnetic data are generally poorly known, the error in the total intensity can be estimated for an average of $11 \mu \mathrm{T}$ and a minimum of $1.5 \mu \mathrm{T}$ (Korte and Constable, 2005a, 2005b). Using these values and assuming that the same relative error for all Gauss coefficients at a given epoch decreased linearly with time from the maximum $33 \%$ at $5000 \mathrm{BC}$ to the minimum $3 \%$ at $1950 \mathrm{AD}$, the errors for the information content have been here estimated by applying the propagation method. These error estimates may be too small, particularly for the period before $2000 \mathrm{BC}$ because the data in this period on which the model was based are sparse and more inaccurate than the later ones. For the $\mathrm{K}$-entropy errors, we took into account both those coming from the propagation method and those associated with the estimates of the slopes of the linear fit over each 100-year interval. In general, the larger errors in the CALS7K model were from the propagation method, while in the IGRF model they were from the linear fit procedure.

Another continuous global geomagnetic field model, called CALS3K (Continuous Archeomagnetic and Lake Sediment model for the past $3 \mathrm{~K}$ years, v.3; time validity: 1000 BC-1990 AD), based on all available archeomagnetic and sedimentary data, has been recently updated with its version 3, that can be considered the best global representation of the past field (Korte et al., 2009). This model was constructed with the same criteria used for CALS7K, except for minor modifications of standard inversion strategies (Korte et al., 2009) implemented, among other reasons, also to have better agreement with GUFM1 model (Jackson et al., 2000). We extended our analysis to the CALS3K model in order to verify the significance of the resulting comparison between IGRF and CALS7K, at least for the past 3000 years. The considerations about the errors previously made for CALS7K can be reasonably applied also to CALS3K analysis, although in the next figures, for visual convenience, we will not show the corresponding errors.

\section{Shannon information comparison}

From the Gauss coefficients of the CALS7K and CALS3K models, we estimated the Shannon information of the geomagnetic field $I(t)$ according to Eq. (2). Although the dating uncertainty of the models is, in average, equal to or greater than 100 years (Korte and Constable, 2005a), we extracted the Gauss coefficients every 5 years for consistency with IGRF that presents its Gauss coefficients at 5-year intervals. This is then reconciled by the successive K-entropy analysis that makes a linear fit at steps of 100 years. We applied definition (2) both for $r=a$, i.e. at Earth's surface as in De Santis et al. (2004), and $r=b=3485 \mathrm{~km}$, i.e. at the Core Mantle Boundary (CMB). We considered also the CMB because it is the place of most of the geomagnetic field sources, thus theoretically more indicative of the dynamical characteristics of the geomagnetic field. We still maintain the analysis at the Earth's surface because of the smaller involved errors and because the models are more comparable at this surface: on the contrary, at the CMB, while CALS7K and CALS3K are regularised so they are quite smooth, this is not the case of IGRF. Figure 1 shows all curves both at $r=a$ and $r=3485 \mathrm{~km}$. For visual convenience, the propagated error bars for CALS7K are shown only every 1500 years while they are not shown for CALS3K, although we expect they are of comparable order with those of CALS7K. In this figure, we also plot the corresponding quantities for the IGRF from 1900 to 2005. The ranges (minimum and maximum values) of $I(t)$ values for all curves, together with their averages, are summarised in Table 1. For simplicity we assumed here the relative error of $1 \%$ in the IGRF Gauss coefficients, 
Table 1. Maximum, mean and minimum values of Shannon Information $I(t)$ for different global models (CALS7K, CALS7K damped whole period and after 2000-, CALS3K, IGRF) at a) Earth's Surface and b) CMB. Bold values are the most representative (minimum values) for the quantity of interest. IGRF $I(t)$ value is the best among all models (except for the values of the most unreliable (older) part of CALS7K before $2000 \mathrm{BC}$ ).

\begin{tabular}{lllll}
\hline a) at Earth's Surface & $I(t)_{\text {CALS7K }}$ & $\begin{array}{l}I(t)_{\text {CALS7K }} \\
(\text { after 2000 BC })\end{array}$ & $I(t)_{\text {CALS3K }}$ & $I(t)_{\text {IGRF }}$ \\
\hline Min Value & $\mathbf{- 0 . 4 3 9 2}$ & $\mathbf{- 0 . 1 3 5 9}$ & $-\mathbf{0 . 2 8 3 0}$ & $\mathbf{- 0 . 3 0 0 4}$ \\
Mean Value & -0.1366 & -0.0927 & -0.1378 & -0.2401 \\
Max Value & -0.0236 & -0.0516 & -0.0772 & -0.1804 \\
\hline b) at CMB & $I(t)_{\text {CALS7K }}$ & $I(t)_{\text {CALS7K (damped) }}$ & $I(t)_{\text {CALS3K }}$ & $I(t)_{\text {IGRF }}$ \\
& & $($ after 2000 BC $)$ & & \\
\hline Min Value & $\mathbf{- 1 . 5 5 7 0}$ & $\mathbf{- 1 . 6 0 6 1}$ & $\mathbf{- 1 . 5 8 7 4}$ & $\mathbf{- 1 . 8 7 8 9}$ \\
Mean Value & -0.7654 & -1.4240 & -1.0568 & -1.7561 \\
Max Value & -0.2626 & -1.1933 & -0.9121 & -1.6518 \\
\hline
\end{tabular}

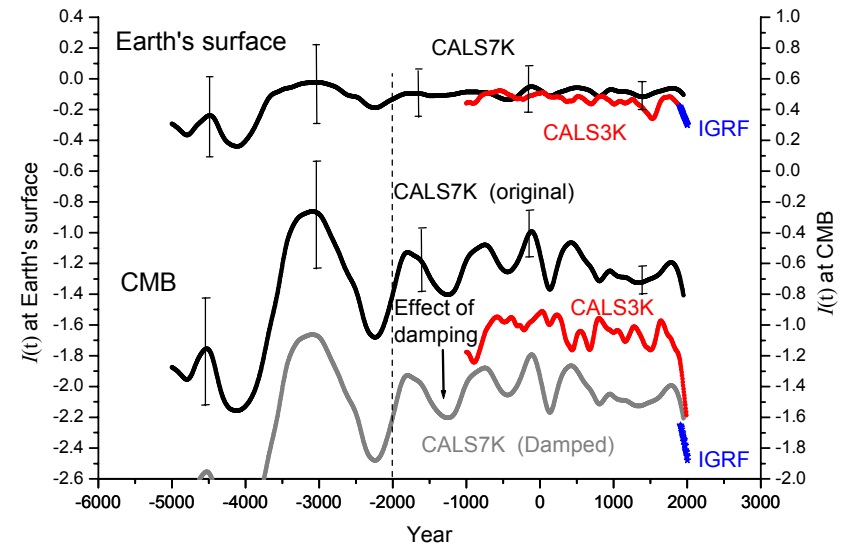

Fig. 1. Shannon Information of the geomagnetic field $I(t)$ from $5000 \mathrm{BC}$ to present (CALS7K - original and damped: 5000 BC-1950 AD; CALS3K: 1000 BC-1990 AD; IGRF: $1900 \mathrm{AD}-2000 \mathrm{AD})$ at the Earth's surface and at the core-mantle boundary $(\mathrm{CMB})$. For visual convenience, propagated error vertical bars (see text) are shown every 1500 years for CALS7K only; although not shown here, the errors associated with CALS3K are comparable. We also show the possible effect of the spectral damping typical of CALS7K model (that we call "CALS7K (damped)") over $I(t)$ at the CMB (at the Earth's surface $I(t)$ is practically unaffected): it allows the "contact" of the corresponding curve (within the estimated errors) with that of IGRF. The curve of CALS3K is intermediate between CALS7K original and damped, suggesting that the damping correction is probably overestimated. The vertical dash line at $2000 \mathrm{BC}$ is drawn as a time reference before which the CALS7K model could not be as reliable as in the successive times (see text).

although more complicated errors could be involved (Lowes, 2000). Please note that, when considering the associated error bars, occasionally the Information content $I(t)$ may show unphysical positive values but which are very small.
This kind of information analysis reveals something more than the usual multipolar approach: in particular, the nondipolar contribution to the (absolute value of the) Shannon information is usually $70-80 \%$ greater than the dipolar one (e.g. Fig. 3 in De Santis et al., 2004; p. 272) confirming that this analysis is really independent from the conventional approach based on the multipolar expansion of the field potential, where the dipole contribution largely dominates all others.

Figure 2 shows the K-entropy of the geomagnetic field as derived applying a linear fit to each 100-year interval at Earth's surface and CMB; each single IGRF value is given in the plots as a star (see also Table 2 for the corresponding mean, maximum and minimum values for each curve). Our results clearly indicate that the Shannon information, after a series of undulations around its average value of almost -0.1 started to decrease at around 1750, so that the present information content reaches a significantly low value of around -0.3 (although this can be also affected by the spectral damping typical of the CALS7K model; please see below), whereas the K-entropy of the field is rather high with respect to the past. Thus, both indicators point to possible chaotic dynamics (e.g., Schuster, 1985) of the processes underlying the geomagnetic field generation in the outer fluid core. This chaotic characteristic of the field surely represents a critical ingredient of the present geomagnetic field (e.g. Barraclough and De Santis, 1997; De Santis et al., 2002, 2004), denoting a global transition possibly going to an imminent excursion or change of polarity. However, besides the worse quality of paleomagnetic and archeomagnetic data, another factor can affect the information content estimation, which is more inherent to the CALS7K construction: the way this model was constrained generates a final damped spatial power spectrum, i.e. the contributions of higher spherical harmonic degrees are strongly reduced with respect to those of the expected real spectrum. In order to quantify the effects of this damping, we 
Table 2. Minimum, mean and maximum values of K-entropy $\mathrm{K}$ for different global models (CALS7K - whole period and after 2000 BC-, CALS3K, IGRF) at a) Earth's Surface and b) CMB. Bold values are the most representative (maximum value) for the quantity of interest. The most recent K-entropy values (CALS3K at 1990 and IGRF) are the best among all models.

\begin{tabular}{lrlll}
\hline a) at Earth's Surface & $K_{\text {CALS7K }}$ & $\begin{array}{l}K_{\text {CALS7K }} \\
\text { (after 2000 BC) }\end{array}$ & $K_{\text {CALS3K }}$ & $K_{\text {IGRF }}$ \\
\hline Min Value & -0.00103 & -0.00043 & -0.00074 & \\
Mean Value & -0.00003 & -0.00001 & 0.00005 & \\
Max Value & $\mathbf{0 . 0 0 1 0 4}$ & $\mathbf{0 . 0 0 0 3 4}$ & $\mathbf{0 . 0 0 1 1 7}$ & $\mathbf{0 . 0 0 1 7 7}$ \\
\hline b) at CMB & $K_{\text {CALS7K }}$ & $\begin{array}{l}K_{\text {CALS7K }} \\
\text { (after 2000 BC) }\end{array}$ & $K_{\text {CALS3K }}$ & $K_{\text {IGRF }}$ \\
& & -0.00182 & -0.00178 & \\
\hline Min Value & -0.00229 & -0.00003 & 0.00015 & \\
Mean Value & -0.00009 & $-0.001590)$ & $\mathbf{0 . 0 0 1 9 4}$ \\
Max Value & $\mathbf{0 . 0 0 1 9 3}$ & $\mathbf{0 . 0 0 1 9 3}$ & $\mathbf{0 . 0 0 3 9 9}$ (at 1990) & \\
\hline
\end{tabular}

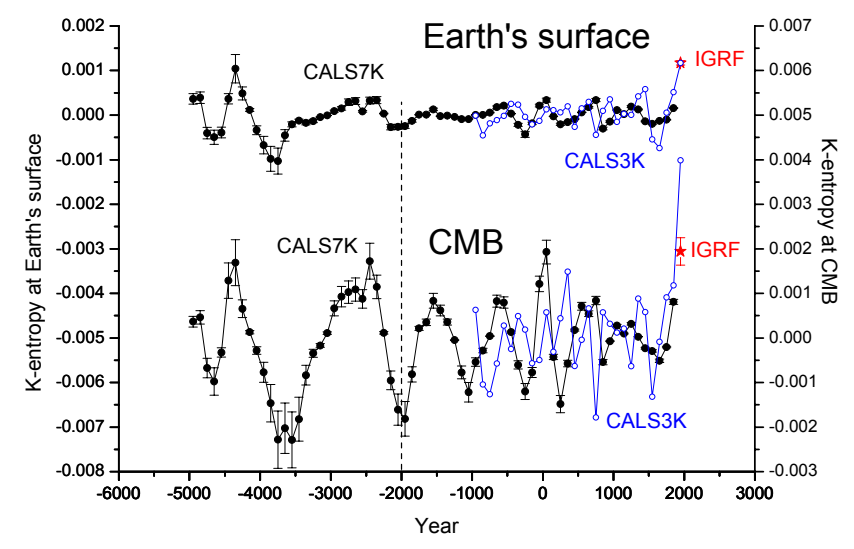

Fig. 2. K-entropy of the field from $5000 \mathrm{BC}$ to present from CALS7K (full circles), CALS3K (empty circles) and IGRF (star) models, at the Earth's surface and at the core-mantle boundary. The present value of K-entropy of the field based on the IGRF model is the highest over all the investigated period (see also Table 2). Before 3500 BC, K-entropy at the Earth's surface shows a large oscillation with highest value at around $4400 \mathrm{BC}$ comparable with the most recent one, while at the $\mathrm{CMB}$ there are other positive peaks at around 0, 2500 and $4400 \mathrm{BC}$ with comparable amplitude. The vertical dash line at $2000 \mathrm{BC}$ is drawn as a time reference before which the CALS7K model could not be as reliable as in the successive times (see text).

try to mimic the situation considering a reasonable spectral correction model for CALS7K in order to be closer to the spectrum of the recent IGRF. Looking at the geomagnetic field power spectra of CALS7K and IGRF in some overlapping epochs (an example is given in Fig. 3 for epoch 1950) we find a good agreement when we multiply the real spectrum of CALS7K by a factor $\exp (-0.33 \cdot(n-1))$. This is also confirmed by the good agreement of the CALS3K power spectrum with both IGRF and the damped CALS7K power spectra. We now look at what happens to the information

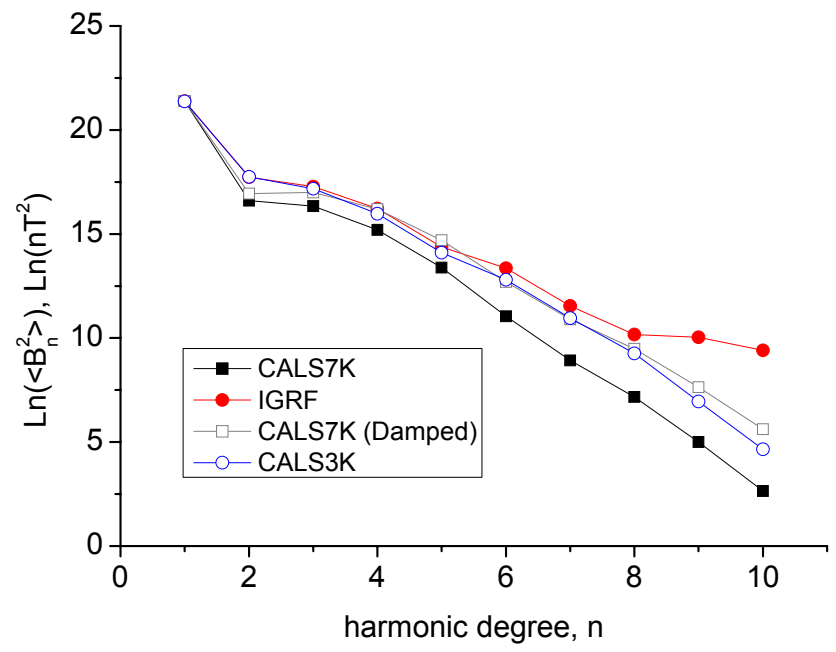

Fig. 3. Spatial power spectra of CALS7K, Damped CALS7K, CALS3K and IGRF at Earth's surface, epoch 1950.

quantities, i.e. we see the main differences (here called $\Delta \varepsilon$ ) "Original Model" - "Damped Model" of the corresponding information quantities: the applied damping generates on average an almost fixed variation in the absolute level of $I(t)$, unaffecting K-entropy estimated from the time derivative of the former quantity. In particular, we find that the greater effect is on $I(t)$ at the CMB, with $\Delta \varepsilon=-0.8$ (with a range of variation of \pm 0.1 ), from a comparison in the epoch 1900 1950 with IGRF, while we do not "correct" the curve at the Earth's surface, because its correction is practically negligible (the estimated correction would have been $\Delta \varepsilon<-0.1$, with a range of variation of \pm 0.05 ). In general, this "adjustment" was such as to make almost contact between the curves from CALS7K and IGRF over the overlapping period of validity. Figure 1 shows also the "adjusted" curve (indicated as "CALS7K (damped)") at CMB obtained adding $\Delta \varepsilon$ 
to the original curve. We recognise that our correction in $I(t)$ is not resolutive, since the two models, CALS7K and IGRF, have been created for different purposes: the former is a global "smoothed" representation of the field with regularisation at the source level, while the latter is a global representation of the present field without constraints or regularisation at any level. For this reason, although we are looking at the complete span of the past 7000 years, we will also compare our results with those obtained from CALS3K analysis over a shorter 3000-year time window. However, we believe that with this correction we are able to make a reasonable comparison of IGRF and CALS7K models in terms of the Shannon information and related quantities, at least for the past 4000 years (see below). After correction, the present values of the information content both at the Earth's surface and at CMB are still the lowest ones since $2000 \mathrm{BC}$ (a vertical dash line identifies this epoch; see also the minimum values for each curve which are bold in Table 1).

Regarding the K-entropy, we see that, in the whole investigated period, its present value (for CALS3K at 1990 and IGRF) at Earth's surface and CMB is the highest (see the maximum values for all curves which are bold in Table 2), although, before around $3500 \mathrm{BC}$ K-entropy at the Earth's surface shows a large oscillation with highest value at around $4400 \mathrm{BC}$ which is comparable with the present one. At the CMB few other positive peaks emerge at around 0,2500 and $4400 \mathrm{BC}$, with comparable values. By the way, an interesting feature of K-entropy, clearer at CMB than at Earth's surface, is the presence of persistent oscillations with periods ranging between 500 and 1600 years, that generally tend to decrease (in time and in amplitude) towards present, with the exception of IGRF which is the highest value. The question whether these features are real characteristics of the geomagnetic field or an artefact of the CALS7K model would deserve deeper investigation in future works.

For all curves, we cannot exclude the possibility that the errors associated with CALS7K, especially for periods before $2000 \mathrm{BC}$, were underestimated because the experimental data on which the model was based were sparse and inaccurate. This is also confirmed by recent studies of this model and its used dataset: for instance, Leonhard and Fabian (2007), excluding the most critical data, i.e. lake sedimentary data, from the original dataset, find a new resulting model with significant differences with respect to CALS7K, especially before $1500 \mathrm{BC}$. On the other hand, a disadvantage of using only archeomagnetic data is that they are geographically sparser than sedimentary data, so that a model based on better, but sparser data is not necessarily an overall better model.

From the Shannon information of CALS3K, which shows an intermediate behaviour at the CMB between the original CALS7K and the damped one, we notice that our damping correction could be overestimated, re-enforcing the impression of a very low information content (and high K-entropy) of the present geomagnetic field.

\section{Discussion and conclusions}

The aim of this paper has been to describe how the present geomagnetic field behaves with respect to the field of recent past, in terms of some more universal quantities, such as the Shannon Information and the Kolmogorov entropy, in order to exploit the dynamical properties of the geomagnetic field in its wholeness. To do this, we have analysed the temporal behaviour of the field's information content and K-entropy as deduced from the CALS7K, CALS3K and latest IGRF models. Tables 1 and 2 show averages and ranges of variations of both quantities, printing in bold the most representative values for each quantity, i.e. the minimum values for $I(t)$ and the maximum vales for K-entropy. Although some undulations in both quantities appear before $2000 \mathrm{BC}$, we find that the information content has been decreasing over most of the recent times, especially in the past 250 years, confirming the analyses of IGRF and GUFM1 models made by De Santis et al. (2004) and De Santis (2007). The highest values of the K-entropy of $\mathbf{B}$ in recent times reflect a geomagnetic field which is more chaotic than the past field since around 1750 , suggesting a possible critical state of the present geomagnetic field (De Santis et al., 2004). This does not mean exclusively the occurrence of an impending geomagnetic reversal or excursion, only that the present state is critical in the sense that its future behaviour is unpredictable, because it could move either to a global geomagnetic change or to more normal conditions, as already happened in the course of the past thousands of years. This is consistent with most of the results of De Santis $(2007,2008)$ who, analysing the past 400 years from the GUFM1 model, found that the information content of the geomagnetic field started to decay between 1700 and 1800, and it is still decaying (De Santis et al., 2004). Other possible recent confirmations of the present transitional state of the geomagnetic field could be the complex dynamics over the past 400 years of the South Atlantic Anomaly (De Santis and Qamili, 2010), a huge depression of the geomagnetic field strength in the Southern Hemisphere that could go to zero by a few hundreds of years, and the recent detection of rapidly changing dynamics in the Earth's fluid core from satellite data with timescales less than 1 year (Olsen and Mandea, 2008).

These results must clearly be viewed with some caution. First, we admit that our spectral correction as determined by simple spectral comparisons between IGRF and CALS7K to reduce the effect of damping on CALS7K is subjective: this is clear when we compare damped CALS7K results with those from CALS3K that show smaller (absolute) Shannon information values at $\mathrm{CMB}$, suggesting a possible overestimation of our correction. Second, our results for times before $2000 \mathrm{BC}$ are especially problematic because of possible artefacts in the CALS7K model, which mainly include a) the considerable errors in the magnetic field total intensity data from archeomagnetic and sediment data, especially in the $\mathrm{BC}$ times. We also assumed a simple time-dependent error 
which is highly subjective because b) the data distribution for the CALS7K model is very sparse, especially in the Southern Hemisphere where the model is poorly constrained, but the evidence for a possible global transition of the geomagnetic field, possibly going to an excursion or even a reversal is becoming increasingly apparent (Gubbins, 1987; De Santis, 2007). Furthermore, c) the geomagnetic field itself often shows very erratic and unexpected changes (Gubbins et al., 2006).

Although the period of time here considered is far from being comparable with those (of the order of thousands or even hundreds of thousands of years) covering a complete transition such as a reversal or an excursion, we think that the study of this part of the process (that can include those features of the secular variation known as jerks, western drift, dipolar field decay and others) can help in the understanding of the complete process of transition from normal to excursion or reversal states (e.g., see also Gubbins, 1994). Of course, we cannot exclude the possibility that the "unusual" behaviour of the present geomagnetic field will become again more "normal", stopping the present general decay.

The forthcoming ESA Swarm mission will consist of three satellites simultaneously recording the geomagnetic field over three different orbits for a significant time (more than 5 years). This unique dataset, together with all available ground and marine data, will provide the way to determine the best geomagnetic models ever. This, in turn, will probably allow a better assessment of the interesting aspects of the present geomagnetic field found and described here.

Acknowledgements. We thank Monika Korte for providing the CALS7K model together with the extracting algorithm and Roman Leonhard for providing preliminary results on his global models based on different selections of the same paleomagnetic dataset. We thank the Editor (Ulrike Feudel) and three anonymous referees for their useful indications. David R. Barraclough, Gauthier Hulot and Ralph Von Frese provided important comments and suggestions to improve a preliminary version of the paper. This work was funded by the Programme of National Research in Antarctica (PNRA) in the framework of the "Reversing Earth Magnetism?" (REM) Project. Part of the work was made in the frame of the Italian-Albanian bilateral project E-MAG.

Edited by: U. Feudel

Reviewed by: three anonymous referees

\section{References}

Baranger, M., Latora, V., and Rapisarda, A.: Time evolution of thermodynamic entropy for conservative and dissipative chaotic maps, Chaos Soliton. Fract., 13, 471-478, 2002.

Beck, C. and Schlögl, F.: Thermodynamics of Chaotic Systems, Cambridge University Press, Cambridge, 306 pp., 1993.

Buchner, T. and Zebrowski, J.: Local entropies as a measure of ordering in discrete maps, Chaos Soliton. Fract., 9(1/2), 19-28, 1998.
Constable, C. G. and Korte, M.: Is Earth's magnetic field reversing?, Earth Planet. Sc. Lett., 246, 1-16, 2006.

De Santis, A.: How persistent is the present trend of the geomagnetic field to decay and, possibly, to reverse?, Phys. Earth Planet. In., 162, 217-226, 2007.

De Santis, A.: Erratum to "How persistent is the present trend of the geomagnetic field to decay and, possibly, to reverse?", Phys. Earth Planet. In., 170, p. 149, 2008.

De Santis, A.: Geosystemics, Proceedings of WSEAS Conference on Geology and Seismology GES '09, Cambridge, 36-40, February 2009.

De Santis, A. and Qamili, E.: Equivalent Monopole Source of the Geomagnetic South Atlantic Anomaly, Pure Appl. Geophys., in press, 2010.

De Santis, A., Falcone, C., and Lowes, F. J.: Remarks on the meansquare values of the geomagnetic field and its components, Ann. Geofis., 38(2), 167-173, 1995.

De Santis, A., Tozzi, R., and Gaya-Piquè, L. R.: Information Content and K-entropy of the Present Geomagnetic Field, Earth Planet. Sc. Lett., 218, 269-275, 2004.

Eckmann, J.-P. and Ruelle, D.: Ergodic theory of chaos and strange attractors, Part I, Rev. Mod. Phys., 57(0.3), 617-654, 1985.

Ferraro, M., Boccignone, G., and Caelli, T.: Entropy-based representation of image information, Pattern Recogn. Lett., 23(12), 1391-1398, 2002.

Gubbins, D.: Mechanism for geomagnetic polarity reversals, Nature, 326, 167-169, 1987.

Gubbins, D.: Geomagnetic polarity reversals: a connection with secular variation and core-mantle interaction?, Rev. Geophys., 32(1), 61-83, 1994.

Gubbins, D., Jones, A. L., and Finlay, C. C.: Fall in Earth's Magnetic Field is erratic, Science, 312, 900-902, 2006.

Holme, R. and Olsen, N.: The spectrum of the magnetic secular variation, in; Earth Observation with CHAMP, edited by: Reigber, C., Luhr, H., Schwintzer, P., and Wickert, J., Springer, New York, 329-334, 2005.

Hulot, G., Eymin, C., Langlais, B., Mandea, M., and Olsen, N.: Small-scale structure of the geodynamo inferred from Øersted and Magsat satellite data, Nature, 416, 620-623, 2002.

Jackson, A., Jonkers, A. R. T., and Walker, M. R.: Four centuries of geomagnetic secular variation from historical records, Philos. T. Roy. Soc. A, 358, 957-990, 2000.

Jacobs, J. A.: Reversals of the Earth's Magnetic Field, 2nd edn., Cambridge Univ. Press, Cambridge, 366 pp., 1994.

Jernigan, M. E. and D'Astous, F.: Entropy-based texture analysis in the spectral frequency domain, IEEE T. Pattern Anal., PAMI6(2), 237-243, 1984.

Korte, M. and Constable, C. G.: Continuous geomagnetic field models for the past 7 millennia: 2. CALS7K, Geochem. Geophy. Geosy., 6, Q02H16, doi:10.1029/2004GC000801, 2005a.

Korte, M. and Constable, C. G.: The geomagnetic dipole moment over the last 7000 years - new results from a global model, Earth Planet. Sc. Lett., 236, 348-358, 2005b.

Korte, M. and Constable, C. G.: Centennial and millennial geomagnetic secular variation, Geophys. J. Int., 167, 43-52, 2006.

Korte, M., Donadini, F., and Constable, C. G.: Geomagnetic field for $0-3 \mathrm{ka}$ : 2. A new series of time-varying global models, Geochem. Geophy. Geosy., 10, Q06008, doi:10.1029/2008GC002297, 2009. 
Leonhardt, R. and Fabian, K.: Geomagnetic variation during the past 5000 years: A global reconstruction, IUGG XXIV General Assembly, Perugia, Italy, poster presentation 2554, ASI003, 2007.

Lowes, F. J.: Mean-square values on sphere of spherical harmonic vector fields, J. Geophys. Res., 71, 2179, 1966.

Lowes, F. J.: Spatial power spectrum of the main geomagnetic field, and extrapolation to the core, Geophys. J. Int., 36, 717-730, 1974.

Lowes, F. J.: An estimate of the errors of the IGRF/DGRF fields 1945-2000, Earth Planets Space, 52, 1207-1211, 2000.

Macmillan, S. and Maus, S.: International Geomagnetic Reference Field - the tenth generation, Earth Planets Space, 57, 1135-1140, 2005.

Majewski, E.: Thermodynamics of Chaos and Fractals applied: Evolution of the Earth and Phase Transformations, in: Earthquake Thermodynamics and Phase Transformations in the Earth's Interior, edited by: Teisseyre, R. and Majewski, E., 2580, 2001.
Olsen, N. and Mandea, M.: Rapidly changing flows in the Earth's core, Nature Geoscience, 1, 390-394, 2008.

Rodrìguez-Iturbe, I., D’Odorico, P., and Rinaldo, A.: Configuration entropy of fractal landscapes, Geophys. Res. Lett., 25(7), 10151018, 1998.

Ryzhikov, G., Biryulina, M., and Hanyga, A.: 3D nonlinear inversion by entropy of image contrast optimization, Nonlin. Processes Geophys., 2, 228-240, 1995, http://www.nonlin-processes-geophys.net/2/228/1995/.

Schuster, H. G.: Deterministic Chaos, VCH, Weinheim, 291 pp., 1995.

Shannon, C.: A mathematical theory of communication, Bell System Technical Journal, 27, 379-423, 623-656, 1948.

Wales, D. J.: Calculating the rate of loss of information from chaotic time series by forecasting, Nature, 350, 485-488, 1991. 